\title{
Research Progress in Preparation Technology of Metal Rubber Blank
}

\author{
Kenan Wu, Hongbai Bai,TuoLi \\ Department of Vehicle and Electrical Engineering, Army Engineering University, Shijiazhuang \\ 050003, China \\ Wukenan333@163.com
}

Keywords: metal rubber; blank ; preparation technology

\begin{abstract}
Metal rubber is a kind of functional structurematerial, which has important application value in military equipment and civil machinery. The preparation process of metal rubber blanks plays a key role in the performance and structure of metal rubber and is the foundation of metal rubber research. In this paper, the research status of metal rubber blank preparation technology is described, and the preparation technology of metal rubber blank is classified and summarized. The development trend and research direction of the preparation process of metal rubber blank are discussed.
\end{abstract}

\section{Introduction}

Metal rubber is a kind of elastic porous material with non-linear stiffness and large damping. The interior of the metal rubber is a space network formed by interlocking metal wires. The metal rubber is resistant to radiation, corrosion, high / low temperature, long fatigue life and vacuum Non-volatile, stable performance, etc., are widely used in cutting-edge weaponry and industrial production. In the field of military applications, metallic rubber has broad application prospects in the fields of unmanned aerial vehicles, submarine photovoltaic masts, torpedoes, space engines, submarine pipelines, and the like, which need to have both damping and cushioning properties and harsh environments. In the civilian sector, metal rubbers are widely used in fields such as Galileo satellite navigation laser reflectors, meridian sounding rockets, industrial pipelines, deep sea submarines, polar rover robots, etc.that require both cushioning and harsh environments [1-4].

The process of preparing metal rubber is to select the material (brand) and diameter of the wire, winding spiral wound, to prepare the blank, the cold stamping and the later treatment.The size, shape, porosity, damping and strength properties of metal rubbers are largely related to the preparation of the blank. The preparation of the metal rubber blank is based on the fact that the metal spiral coil is closely connected, the metal spiral coil space is evenly distributed and the density is constant, and the amount of the helical coil is less, the porosity of the metal rubber is as small as possible. According to the preparation principle and preparation equipment of metal rubber blank, the preparation technology of metal rubber blank is divided into hand-made paving blank technology, spiral wound blank technology, metal mesh blank weaving technology, CNC spiral blank laying blank technology and some special technologies.

\section{Hand-made paving blank technology}

Metal rubber research initially, due to the preparation conditions, most of the way by hand-made metal rubber preparation. The Russian National Samara University of Aeronautics and Astronautics has done a lot of research in the field of hand-laid blank technology. According to the structure and performance of metal-rubber under different application environments, many preparative processes have been designed.For example, in order to expand the application range of metal rubber damping performance and tensile strength, a process of preparing a blank by winding a spiral coil and a thin wire harness is designed. The damping performance and strength of metal rubber can be improved by adjusting the diameter and specific gravity of the filament, and the pitch of the spiral coil. In order to improve the strength of the metal rubber shock absorber, a method of winding and interlacing the 
spiral coil to the metal wire Rubber blank. The technology improves the strength of the shock absorber while ensuring the frequency characteristics of the shock absorber. In order to improve the service life of the metal rubber and the stability of the damping property of the metal rubber, a layered blank laying method is used. In the first layer, the spiral coils are laid alternately and the second spiral coils are laid in parallel. The third layer is laid in the same way as the first layer. In order to improve the damping capacity of metal rubber and reduce the resonance amplitude of it, the design of spiral winding are stacked into a similar blanket shape blank preparation technology of [5].

In the current research stage, some researchers still use hand-paved methods to prepare metal rubber blank in order to prepare some special-purpose metal rubbers.In order to improve the degree of biting of metal spiral coils, Wang Fengming designed the ring overlapping network, well pattern laying and cross hook networking of three kinds of spiral wound weaving method of metal rubber blank.At the same time, the process also improved the uniformity of material [6].Wang Ruirui task force will be the spiral metal spiral in accordance with the "well" shape two-dimensional blank laying, and then the two-dimensional blank rolled into a three-dimensional blank, the preparation process to improve the smoothness of hand-woven and reduce the difficulty of weaving [7];Firstly, a two-dimensional blank is woven by spiral rolling and then a two-dimensional blank is wound to form a three-dimensional blank. Dong Xiuping is based on the preparation process of the blank, based on the assumption of two-dimensional uniformity, a numerical model of cylindrical metallic rubber is established. Metal-like rubber products provide theoretical support for the design and preparation [8]; In order to make metal rubber more uniform density, more stable performance, Yang Kunpeng the spiral wire in accordance with the ratio of 2, 1 is divided into two, first of the specific gravity of 2 spiral wound into a ribbon, and its along the mandrel Winding to form the preliminary blank. Then the specific gravity of 1 wound on the bobbin, making the blank more tight, no scratches the surface of metal rubber to overcome the uneven density of metal rubber and easily delaminated shortcomings [9].

These innovative blank preparation processes expand the field of application of metallic rubbers and offer a wealth of ideas for mechanized development of metallic rubber blank preparation processes. However, hand-made paving technology, complex technology, preparation efficiency is not high, the technical requirements of the preparation staff is relatively high, and metal-rubber product structure parameters and performance of the relatively poor, can not meet the needs of largescale modern defense industry.

\section{Spiral wound blank technology}

In the preparation of conventional size and configuration of simple metal rubber products, often used spiral wound blank technology to prepare metal rubber blank. In order to improve the mechanization level of metal rubber preparation, Li Mingsen selected single-chip controlled stepper motor as the power unit, developed a movable spiral coil laying platform to achieve the laying of metal rubber blank. On the basis of the automatic spiral coil laying platform, the advantages and disadvantages of two different winding modes of fine needle positioning and pressure line positioning are studied. It is proposed that the fine needle positioning winding method, the spiral coil distribution rule, the higher the degree of wire meshing, the smaller the size and the higher performance metal rubber products, but the efficiency is lower and the production cost is higher. The pressure line positioning winding method, the operation is relatively simple, easy to adjust the height of the rough laying, suitable for larger size, less demanding on the performance of metal rubber products [10]; Chen Hui, Zhu Yuquan and other rubber needle localization with metal blank winding principle as the foundation, through the movement characteristics of the fine needle positioning winding and research methods of fine needle positioning winding, design a new type of winding equipment, to achieve the display and control winding movement parameters and regulation of [1112].

Spiral winding blank preparation process proposed, making the metal rubber product yield has been improved, a substantial increase in production efficiency, significantly reduce production costs. Bai Hongbai research group topics in order to reduce the spiral wound in the process of winding the 
blank to improve the metal rubber molding size and performance consistency. A CNC spiral winding machine based on PMAC multi-axis control technology was developed(Fig. 1), which realized the fully automatic precise control of the blank winding from the helical winding to the blank winding. At the same time, Wire diameter and coil diameter is unlimited, suitable for the preparation of conventional size and configuration simple metal rubber products. In the actual production has achieved good results, is the most common metal and rubber blank preparation process, the most stable preparation process.

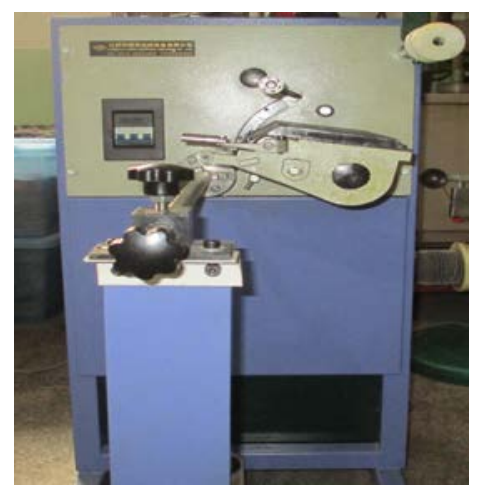

Figure .1 CNC spiral wound winding equipment

\section{Metal mesh blank weaving technology}

Metal wire mesh weaving blank technology developed in recent years is a new type of metal rubber blank preparation process, with the progress of the research, the technology gradually mature. The basic process, the metal wire braided braided according to certain rules by metal wire mesh weaving equipment, and then the metal mesh for certain processing finally, the metal wire wound into a metal rubber blank.

In order to solve the problem of small density, forming the stiffness of metal rubber. Li Tuo reference preparation process of knitted material, using circular weft knitting machine (Fig. 2) the metal wire woven into a net, and then use the pressure tank equipment (Fig. 3) the net pressure tank, winding finally, stamping molding, prepared into a braided - block groove metal rubber wire braid NC groove blank, high degree of automation, low scrap rate, the size and performance of metal rubber prepared. Through the experiment, the preparation technology of metal rubber, vibration a narrow frequency band, and in the process of using small residual deformation structure and stable performance Good sex, long service life [13].

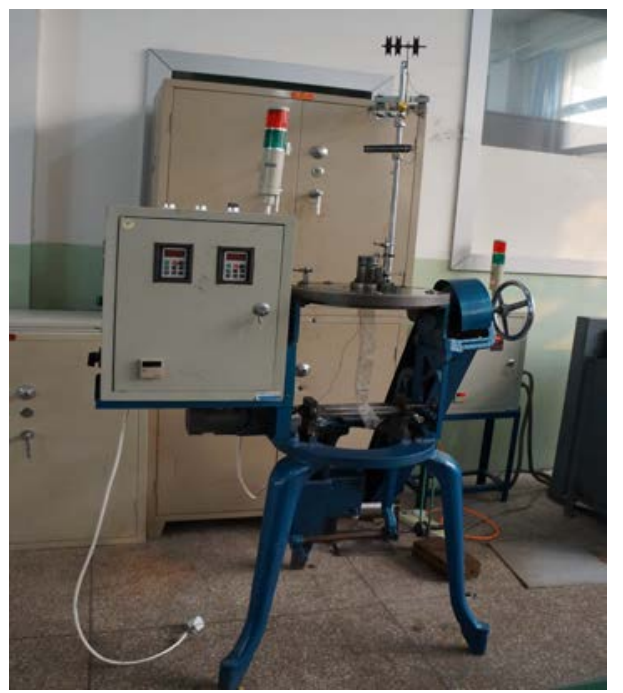

Figure.2 Metal wire mesh weaving equipment 


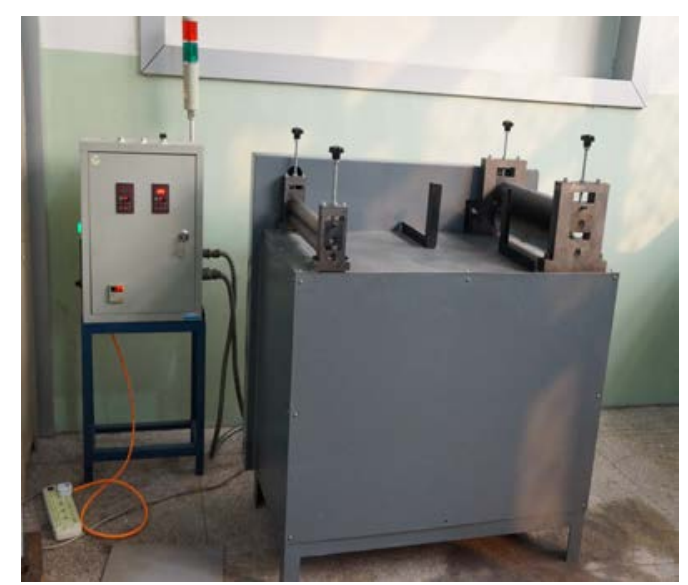

Figure .3 wire groove pressing equipment

Zou Guangping independently developed a metal mesh rubber preparation process, the process for the first use of metal wire mesh weaving machine, the metal wire woven into the net, and then the forming of metal wire mesh weaving lines suppressed, which made of metal rubber blank. The process of high degree of mechanization, high production efficiency, is conducive to scale the metal wire mesh production. Metal rubber static compression, shear, swept sine and random vibration test, put forward, rubber damping characteristics of metal wire mesh and the mechanical properties of the prepared more stable [14-16].He Weiming, Ye Wenbo reference fabric flat knitting technology of metal wire warping after made spiral, and then through the hook weaving process will spiral woven metal mesh, finally the metal mesh winding into the metal rubber blank, the hook weaving technique ensure repeat unit regularity, strengthen the stability of the [17-18]. performance metal rubber.

\section{CNC spiral blank laying blank technology}

In order to obtain metal rubber articles with special properties and complex configurations, handweaving methods are often used to prepare metal rubbers. But the manual weaving route is basically based on experience, and the preparation efficiency is not high. The technical requirements for the preparation personnel are relatively high, and the stability of metal rubber products is difficult to guarantee.In order to improve the science of weaving path and the stability of the performance of metal rubber, Bai Hongbai research group developed CNC blank laying equipment based on the PMAC multi-axis control technology (Fig. 4), achieving helical winding pitch stretching, threeLaying track planning and three-dimensional laying rough automatic precision control. As the weaving path can be precisely controlled, the prepared metal rubber, performance and size specifications more in line with design requirements.

CNC spiral blank laying blank technology effectively solves the problems of large size metal rubber sheet and metal rubber preparation problems with complex geometries. The technical difficulties of the process, design optimization of blank laying path, the path planning into the moving path of the manipulator. Huang Kai of the metal rubber blank automatic laying equipment and process to conduct the thorough research, put forward the feasible path blank laying strategy, at the same time with the background grid technology as the foundation, established the positioning pin design and planning and design method of laying path using genetic algorithm, improves the metal rubber blank uniformity and density, provides a theoretical basis for the laying of blank [19 -21]. 


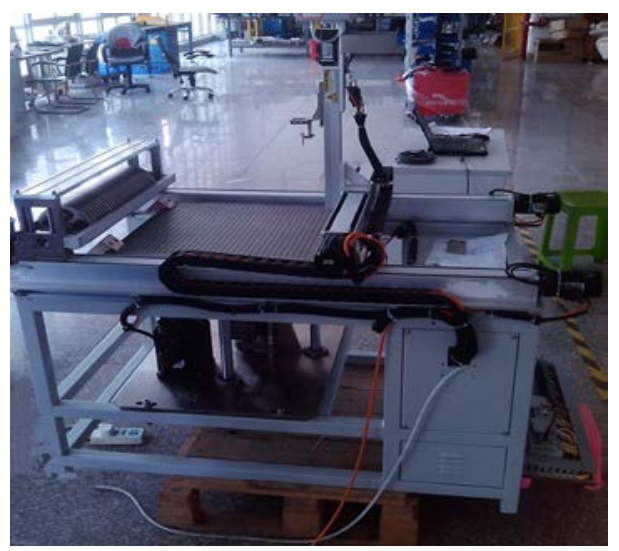

Figure.4 CNC laying rough paving equipment

\section{Preparation of other blanks}

Some researchers, starting from the preparation materials and preparation methods of metal rubber, have innovated the preparation of metal rubber and enriched the diversity of metal and rubber products.

Mao Chenxi use memory alloy wire preparation of metal rubber, pointed out that with the advantages of ordinary metal rubber, but also have good shape recovery controllability [22],Huang Rui studied the mechanical properties of the new metal rubber. Based on the experimental results, the change rule of equivalent damping and equivalent stiffness of metal rubber was analyzed [23].Zhao Cheng used 65Mn preparation of large diameter steel wire and large diameter spiral, spiral coil made of two dimensional crisscross mesh, several mesh stacked along the vertical mesh screwed into the spiral coil to be fixed, and finally use the metal stamping . The metal rubber prepared by this raw material and process can be adapted to large load conditions [24]; Chen Ya proposed a high-energy pulse discharge system of metal rubber material discharge sintering, and passed the experimental verification [25].

\section{Conclusion}

Metal rubber preparation process is always accompanied by a metal rubber application background and research needs of development, with the research of metal rubber constantly, improve the technological level of the continuous preparation of metal rubber, metal rubber structure more reasonable, the performance is more stable. In the new process for the preparation of background, preparation of metal rubber gradually toward mechanization, automation, application of metal rubber is more widely used.

However, because of the metal rubber preparation technology level, preparation process of metal rubber products, product performance parameters is still no uniform industry standards, restrictions on research and application of metal rubber to a certain extent. In the field of preparation of metal rubber, there are some gaps, such as thin-walled cylindrical shaped, large length diameter ratio study, large size flat type metal rubber preparation technology is still relatively lacking, the research work for further research.

\section{References}

[1] H. B. Bai, C. H. Lu, F. L. Cao, "Metal rubber material and engineering application,” 1sd ed.,Beijing, Science Press,2014,pp.1-27.

[2] J. C. Yang, G. Q. Liu, H. B. Bai, Y. Y. Wang, "Study on Figuration Techniques of Grades Extrusion for Making Metal Rubber Material ", Advanced Materials Research, Vols. 97-101, pp. 1709-1712, 2010 
[3] X. P. Dong and M. J. Huang, "Load Effect on 1Cr18Ni9 Stainless Steel Wires' Fretting Behaviors in Metal Rubber Dampers", Applied Mechanics and Materials, Vols. 291-294, pp. 2744-2748, 2013

[4] Z. Lin, G. Z. L, H. B. Bai, C. H. Lu, "Experimental Investigation on Damping Characteristic of Metal Rubber Material at Simulated Marine Environment", Applied Mechanics and Materials, Vol. 456, pp. 110-114, 2014

[5] Chegodae D, “The design of metallic rubber component,”Translated by Li Z Y. Beijing, National Deference Industry Press, 2000,pp.12-21 .

[6] F. M. Wang, "Metal rubber component preparation, dynamic analysis and vibration characteristics of the theoretical and Experimental Research on," Shijiazhuang,Ordnance Engineering College, 2010 .

[7] R. R. Wang, "Experimental study on static and dynamic mechanical properties of metal mesh rubber damping material,” Harbin Engineering University, 2012 .

[8] X. P. Dong, G. Q. Liu, J. C. Yang, X. Y. Li, "Study on three dimensional geometric model of a new type of metal rubber filter element with adjustable porosity,” Journal of Jiamusi University (NATURAL SCIENCE EDITION), 26(03), 292-295, 2008.

[9] K. P. Yang, "Nonlinear rubber isolator nonlinear characteristics of the study and parameter identification ,” North University, 2017.

[10] M. S. Li, W. Q.Zhang, X. Y. Wu, "Metal rubber automatic molding process research,” Machinery Manufacturing, 537 (47), 55-57, 2009.

[11] H. Chen, "Metal rubber winding forming process and equipment research,” Xi'an University of Technology, 2014 .

[12] Y. Q. Zhu, L. B. Zhou, B. J .Ma, “Metal rubber blank automatic laying process research,” Mechanical Research and Application, 2 (29), 78-80, 2016.

[13] T. Li, H. B. Bai, C. H. Lu, F. L. Cao, "Preparation and Experimental Study on WeavingGroove Metal Rubber,” Mechanical Science and Technology, 34 (03), 481-484, 2015.

[14] G. P. Zhou, Z. Liu, Z. L.Chang, Y. L. Li, “Journal of Experimental Mechanics,” 06, 676682, 2014.

[15] G. P. Zhou, Z. Liu, Z. L.Chang, Y. L. Li, “Journal of Aeronautics and Astronautics,” 10, 2318-2324, 2016.

[16] G. P. Zou, H. Z. Cheng, "Chang Zhongliang, Wang Peng, Wang Ruirui, Study on Static Mechanical Properties of Wire Meshed Metal Rubber," Journal of Harbin Engineering University, 2(03), 332-336, 2015.

[17] W. M. He, "Metal rubber damper network block molding process and performance research,” North University, 2015 .

[18] W. B. Ye, "Metal wire mesh shock absorber static and dynamic characteristics of the test and theoretical research,” North University, 2017 .

[19] K. Huang, H. B. Bai, C. H. Lu, F. L. Cao, “ Metal rubber blank 3D automatic laying trajectory generation method,” Hot processing technology, 45 (24), 57-61, 2016.

[20] K. Huang, H. B. Bai, C. H. Lu, F. L. Cao, "Metal rubber automatic laying process and path discrimination strategy,” Modern Manufacturing Engineering, 02, 126-129 +133, 2016 .

[21] K. Huang, H. B. Bai, C. H. Lu, F. L. Cao, "Metal rubber blank laying path planning," Journal of Aeronautics and Astronautics, 31 (01), 40-48, 2016.

[22] C. X. Mao, S. H. Li, Y. G. B. Zhao, “A new kind of isolation material, shape memory alloy 
metal rubber,” Journal of Civil Engineering, 45 (24), 177-1811, 2010.

[23] R. Huang, "Study on mechanical properties of superelastic shape memory alloy metal rubber,” Shandong Agricultural University, 2016.

[24] C. Zhao, J. Zhang, H. Zhang, "Large diameter 65Mn metal rubber rare earth boron carbonitriding,” Metal heat treatment, 2 (34), 89-9, 2011.

[25] Chen Ya, Bai Hongbai, Lu Chunhong, Metal-rubber high-energy pulsed discharge system based on resistance principle [J]. Modern Manufacturing Engineering, 02, 143-146, 2016. 